\title{
Atmospheric Thermal and Dynamic Vertical Structures of Summer Hourly Precipitation in Jiulong of the Tibetan Plateau
}

\author{
Yonglan Tang ${ }^{1}$, Guirong $\mathrm{Xu}^{1, *}$, Rong Wan ${ }^{1}$, Xiaofang Wang ${ }^{1}$, Junchao Wang ${ }^{1}$ and Ping $\mathrm{Li}^{2}$ \\ 1 Hubei Key Laboratory for Heavy Rain Monitoring and Warning Research, Institute of Heavy Rain, China \\ Meteorological Administration, Wuhan 430205, China; tayola@163.com (Y.T.); wanrong@whihr.com.cn (R.W.); \\ tiaotiao98@163.com (X.W.); nanxingong@163.com (J.W.) \\ 2 Heavy Rain and Drought-Flood Disasters in Plateau and Basin Key Laboratory of Sichuan Province, Institute \\ of Plateau Meteorology, China Meteorological Administration, Chengdu 610072, China; kadi9110@sina.com \\ * Correspondence: grxu@whihr.com.cn; Tel.: +86-27-8180-4913
}

check for

updates

Citation: Tang, Y.; Xu, G.; Wan, R.; Wang, X.; Wang, J.; Li, P. Atmospheric Thermal and Dynamic Vertical Structures of Summer Hourly Precipitation in Jiulong of the Tibetan Plateau. Atmosphere 2021, 12, 505. https://doi.org/10.3390/ atmos12040505

Academic Editor: Maya

Garcia-Comas

Received: 10 March 2021

Accepted: 14 April 2021

Published: 16 April 2021

Publisher's Note: MDPI stays neutral with regard to jurisdictional claims in published maps and institutional affiliations.

Copyright: (C) 2021 by the authors Licensee MDPI, Basel, Switzerland. This article is an open access article distributed under the terms and conditions of the Creative Commons Attribution (CC BY) license (https:/ / creativecommons.org/licenses/by/ $4.0 /)$.

\begin{abstract}
It is an important to study atmospheric thermal and dynamic vertical structures over the Tibetan Plateau (TP) and their impact on precipitation by using long-term observation at representative stations. This study exhibits the observational facts of summer precipitation variation on subdiurnal scale and its atmospheric thermal and dynamic vertical structures over the TP with hourly precipitation and intensive soundings in Jiulong during 2013-2020. It is found that precipitation amount and frequency are low in the daytime and high in the nighttime, and hourly precipitation greater than $1 \mathrm{~mm}$ mostly occurs at nighttime. Weak precipitation during the daytime may be caused by air advection, and strong precipitation at nighttime may be closely related with air convection. Both humidity and wind speed profiles show obvious fluctuation when precipitation occurs, and the greater the precipitation intensity, the larger the fluctuation. Moreover, the fluctuation of wind speed is small in the morning, large at noon and largest at night, presenting a similar diurnal cycle to that of convective activity over the TP, which is conductive to nighttime precipitation. Additionally, the inverse layer is accompanied by the inverse humidity layer, and wind speed presents multi-peaks distribution in its vertical structure. Both of these are closely related with the underlying surface and topography of Jiulong. More studies on physical mechanism and numerical simulation are necessary for better understanding the atmospheric phenomenon over the TP.
\end{abstract}

Keywords: Tibetan Plateau; summer hourly precipitation; thermal and dynamic vertical structure; intensive radiosonde observation

\section{Introduction}

The Tibetan Plateau (TP), known as the "roof of the world" and "the third pole," is the highest plateau in the world with an average altitude higher than $4000 \mathrm{~m}$. Its high terrain can reach the middle troposphere and has a stronger effect on atmosphere than that of plain area. The thermal and dynamic effects of the TP gradually affect free atmosphere through the atmospheric boundary layer (ABL), and result in a significant impact on the weather and climate in China and even the global atmospheric circulation and climate [1-4]. Therefore, the thermal and dynamic effects of the TP on weather and climate attract the attention of meteorological researchers. The atmospheric thermal and dynamic vertical structures over the TP affect the occurrence, development and eastward movement of the low value system in this region, which frequently leads to precipitation over the TP and downstream areas such as the Yangtze River (YR) basin [5-8]. Based on the hourly geostationary meteorological satellite (GMS) infrared brightness temperature observation and the sounding data of five stations along the YR, Zhuo et al. [9] studied a heavy precipitation process caused by the eastward moving convective cloud cluster over the TP in 1998, and pointed out that the main causes of the heavy precipitation were the occurrence, development and eastward movement of the wet core cyclone over 
the TP. This is promoted by the divergence of upper air flow and the convergence of lower air flow, as well as the guidance of high and low level jet and the instability of atmospheric stratification in the troposphere. Zheng et al. [10] analyzed the development and movement process of a TP vortex in late July 2008, and found that adiabatic heating and vertical shear of horizontal wind not only affected the development of the vortex, but also affected the moving direction of the vortex, bringing heavy precipitation to the Sichuan Basin and the middle and lower reaches of the YR. With analysis of MERRA2 (Modern-Era Retrospective Analysis for Research and Applications) and TRMM (Tropical Rainfall Measurement Mission) data, Ma et al. [11] indicated that when the vortex center moves to the eastern TP, there will be strong precipitation near the center and the plateau vortex will further develop. As the vortex system continues to move eastward out of the plateau, the large-scale dynamic background of potential vortex advection increases with height in the middle and lower reaches of the YR, which leads to the development of ascending motion and heavy rainfall.

The above studies show that the atmospheric thermal and dynamic vertical structures over the TP directly affect the occurrence, development and eastward movement of the low value system in this region and play an important role in revealing the formation mechanism and trigger mechanism of precipitation over the TP and its downstream areas. At present, the knowledge of atmospheric thermal and dynamic vertical structures over the TP and their impact on precipitation is still insufficient because of the lack of atmospheric profile observation in this region. Due to the complex terrain and harsh environment of the TP, the observation stations are sparse, and the cost of observation is expensive, so it is difficult to carry out massive, large-area atmospheric observation experiments frequently in this region. Therefore, it is important to study atmospheric thermal and dynamic vertical structures over the TP and their impact on precipitation by conducting long-term observational experiments at representative stations. Since 1978, China has successively carried out several large-scale comprehensive observational experiments in the TP and obtained a large number of valuable observational data. Many scientific research achievements have been made based on these datasets [12-15]. Among these studies, there are relatively few on atmospheric thermal and dynamic vertical structures of precipitation over the TP, especially in different precipitation grades. Since precipitation is highly sensitive to atmospheric thermal and dynamic factors [16], it is of great scientific significance to study the characteristics of atmospheric thermal and dynamic vertical structures in different precipitation grades over the TP, to reveal the formation and trigger mechanisms of precipitation in this region.

The intensive observational experiment of the southwest vortex is an atmospheric science experiment conducted by the Chengdu Institute of Plateau Meteorology of China Meteorological Administration since 2010, aiming to obtain the long-term comprehensive data of radiosonde and ground observations in the experimental area [17]. It is carried out from June to July and lasts 41 days each year in the eastern slope of the TP and the Sichuan Basin. As a main source of southwest vortex, Jiulong County is a key area for the study of TP weather and climate, thus Jiulong is an important station for intensive observational experiments of the southwest vortex [18]. In this study, based on the intensive radiosonde observation of Jiulong from 2013 to 2020 and hourly precipitation data in the same period, the characteristics of precipitation on a subdiurnal scale is analyzed, and vertical structures of atmospheric temperature, humidity and horizontal wind in different precipitation grades are also investigated, in order to provide observational facts for exploring how the thermal and dynamic effects of the TP affect the occurrence and development of precipitation.

Compared to previous studies, the main contributions of this paper are as follows: (1) Precipitation amount and frequency are low in daytime and high in nighttime, and hourly precipitation above $1 \mathrm{~mm}$ mostly occurs at night; (2) Humidity and wind speed profiles fluctuate under precipitation condition and the fluctuation increases with precipitation intensity; (3) Wind speed fluctuation under precipitation is small in the morning, large at noon and larger at night, similar to the diurnal cycle of convective activity over the 
TP; (4) Inverse layer is accompanied by inverse humidity layer, and wind speed presents multi-peaks distribution in its vertical structure.

This paper is organized as follows. Section 2 introduces the materials and methods. Section 3 describes the characteristics of summer hourly precipitation and shows the atmospheric thermal and dynamic vertical structures in different precipitation grades in Jiulong. Section 4 discusses the results and highlights future research works. Section 5 draws the main conclusions of this study.

\section{Materials and Methods}

Jiulong County is located in the eastern TP slope and in the transition zone between the main body of the TP and Chengdu Plain. It has undulating terrain, high in the north and low in the south, with a large elevation difference. Mountains and valleys are the two main geomorphic features in the territory of Jiulong. This unique geographical location makes Jiulong a main source of the southwest vortex and an eastward movement route of the TP low value system $[17,18]$. The Jiulong observation station $\left(101.52^{\circ} \mathrm{E}, 29.02^{\circ} \mathrm{N}\right.$, $2925 \mathrm{~m}$ above sea level) is located on the low mountain side of the northwest-southeast river valley. The two sides with range less than $5 \mathrm{~km}$ away from the observation station are high mountains with altitudes of about $4500 \mathrm{~m}$ (Figure 1).

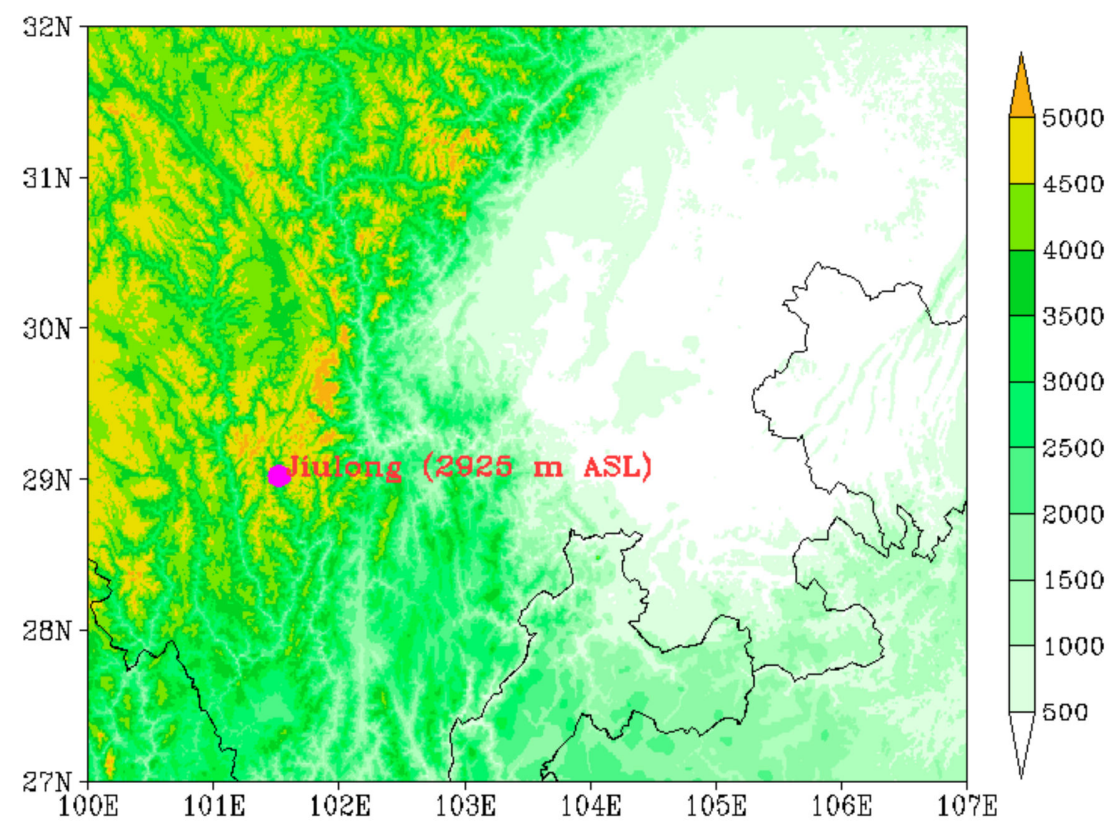

Figure 1. Location of the Jiulong station and its surrounding terrain.

In this study, the intensive sounding and hourly precipitation data of the Jiulong station during the summer of 2013-2020 were used. The intensive sounding data are from the intensive observation experiment of the southwest vortex, which is conducted by the Chengdu Institute of Plateau Meteorology of China Meteorological Administration from 21 June to 31 July (41 days) every year. The GPS (RS41) sounding system manufactured by Vaisala Company of Finland was adapted to measure atmospheric temperature, humidity and horizontal wind profiles in the observational experiment. These sounding profiles were transmitted once per second with a vertical interval of approximately $10 \mathrm{~m}$. According to the operational observation specification, four soundings were launched at 02, 08, 14 and 20 Local Standard Time (LST) each day during the observational experiment [17]. The vertical resolution of the sounding profile was about $10 \mathrm{~m}$ and not fixed, therefore the convenience of statistical analysis and the normalization of all sounding profiles were interpolated with a vertical interval of $10 \mathrm{~m}$. The hourly precipitation data is from the National Meteorological Center of China, from the period of 1 June to 31 August throughout 2013-2020. 
Referring to the methods of Steiner et al. [19] and Zheng et al. [20] and considering hourly precipitation greater than $10 \mathrm{~mm}$ is less in the TP, this study defines four hourly precipitation standards, namely $0.1 \mathrm{~mm}, 0.5 \mathrm{~mm}, 1 \mathrm{~mm}$ and $5 \mathrm{~mm}$, to divide the hourly precipitation grades. This allows for investigation of the characteristics of hourly precipitation in different grades and their corresponding atmospheric thermal and dynamic vertical structures.

To study the diurnal variation of precipitation, the amount, frequency and intensity of precipitation were classified according to different hours of the day $(0-24 \mathrm{~h})$. The amount, frequency and intensity of precipitation at the $24 \mathrm{~h}$ mark were accumulated and dimensionless processing was performed [16,21]. With this method, the diurnal variations of the precipitation amount, frequency and intensity in different grades can be displayed in the same ordinate, which is convenient for comparative analysis. Moreover, in order to analyze the proportion of precipitation in different grades, the precipitation probability is defined as the ratio of the precipitation frequency in a certain grade to the precipitation frequency in all grades, and the precipitation proportion is the ratio of the precipitation amount in a certain grade to the precipitation amount in all grades.

\section{Results}

\subsection{Characteristics of Summer Hourly Precipitation}

\subsubsection{Diurnal Variation}

Diurnal variation of precipitation is a result reflecting the comprehensive influence of atmospheric thermal and dynamic processes on the water cycle [16]. Studying the diurnal variation of precipitation over the TP is helpful not only to understand the characteristics of precipitation on subdiurnal scale, but also to explore the corresponding atmospheric thermal and dynamic processes, and to further understand the formation and evolution mechanisms of precipitation over the TP. Figure 2 shows the diurnal variations of precipitation amount, frequency and intensity in different grades obtained from summer (June to August) of hourly precipitation in Jiulong during 2013-2020. Precipitation amount, frequency and intensity present obvious diurnal variations, especially in the two grades of $[1,5) \mathrm{mm}$ and $[5,10) \mathrm{mm}$. Both precipitation amount and frequency present a similar unimodal pattern in diurnal variation, in which the value is low during the day and high at night with a maximum of 22 LST. For precipitation intensity, although it is generally low during the day and high at night, a bimodal pattern was found for hourly precipitation greater than $1 \mathrm{~mm}$, with the main peak at 22 LST and the second peak at 09 LST. These results indicate that summer precipitation in Jiulong mainly occurs at night, and precipitation amount and frequency are greater at night than during the day. This phenomenon in Jiulong may be related to the effect of valley wind caused by local terrain forcing, because the ascending branch of night valley wind circulation is conducive to the occurrence and enhancement of local precipitation [22].

\subsubsection{Precipitation Probability and Proportion}

The soundings in Jiulong are only launched at 02, 08, 14 and 20 LST each day during the observational experiment ( 21 June to 31 July). Therefore, in order to investigate atmospheric thermal and dynamic vertical structures under precipitation conditions by using sounding profiles, the precipitation probability and proportion in different grades obtained from hourly precipitation at launch times in Jiulong during 2013-2020 were analyzed. As shown in Figure 3, hourly precipitation less than $1 \mathrm{~mm}$ can occur at all launch times and those greater than $1 \mathrm{~mm}$ only occur at 02 and 20 LST. For hourly precipitation less than 1 $\mathrm{mm}$, the precipitation probability is generally higher during the day and the precipitation proportion is more obvious. The maximum precipitation probabilities for hourly precipitation of $[0.1,0.5) \mathrm{mm},[0.5,1) \mathrm{mm},[1,5) \mathrm{mm}$ and $[5,10) \mathrm{mm}$ occur at 14, 08, 20 and 02 LST, respectively, with corresponding values of $88 \%, 33 \%, 32 \%$ and $9 \%$. Moreover, the maximum precipitation proportions also occur at the same launch time for the maximum precipitation probability, with values of $68 \%, 59 \%, 42 \%$ and $80 \%$ for hourly precipitation of 
$[0.1,0.5) \mathrm{mm},[0.5,1) \mathrm{mm},[1,5) \mathrm{mm}$ and $[5,10) \mathrm{mm}$, respectively. Note that although the precipitation probability of hourly precipitation within $1 \mathrm{~mm}$ is higher than that of hourly precipitation above $1 \mathrm{~mm}$, the precipitation proportion of the latter is far higher than that of the former. During the observational experiment period in Jiulong, hourly precipitation of less than $1 \mathrm{~mm}$ can occur during the day, while hourly precipitation greater than $1 \mathrm{~mm}$ mostly occurs at night and is the main contributor to the nighttime precipitation amount.
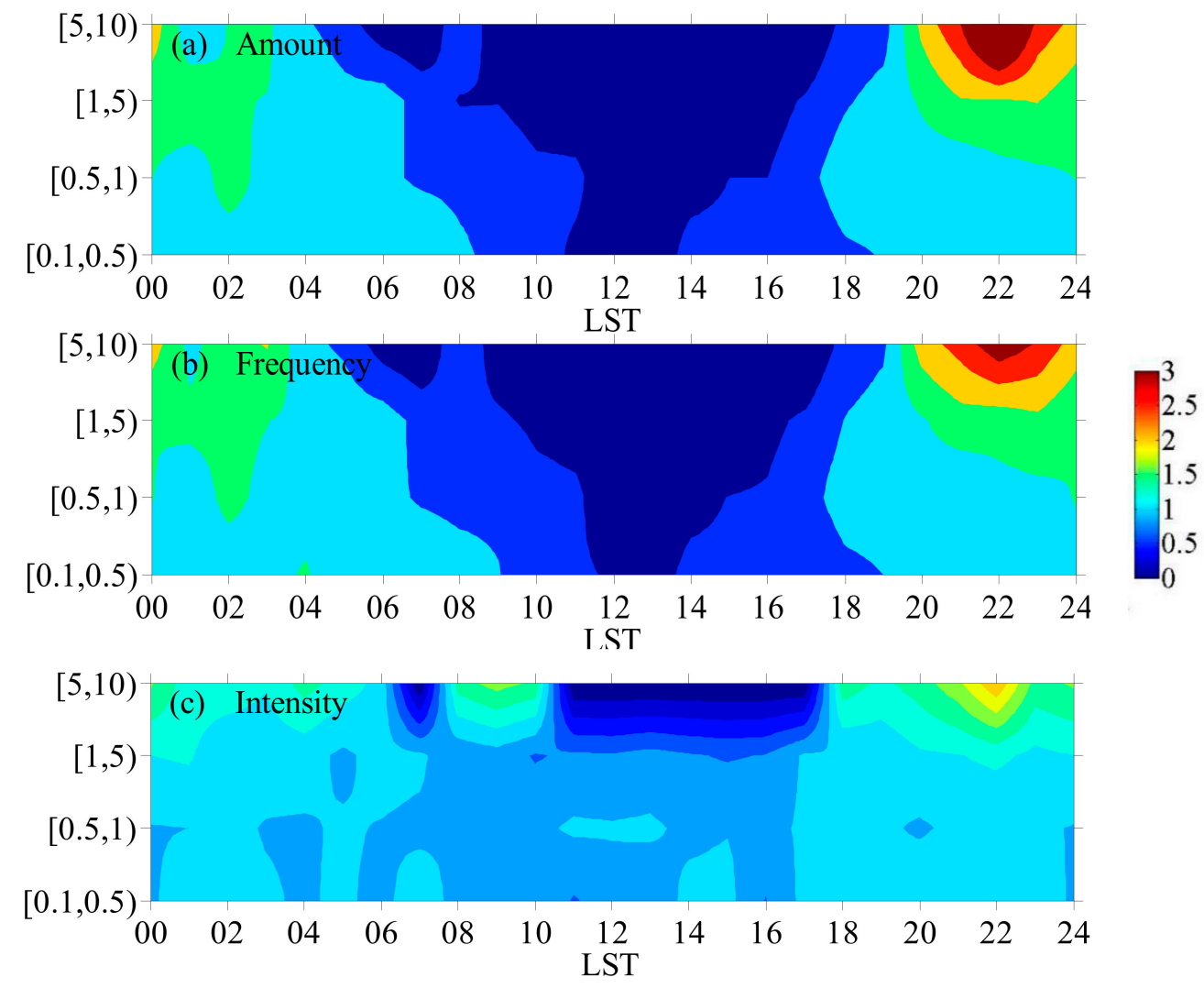

Figure 2. Diurnal variations of precipitation (a) amount, (b) frequency and (c) intensity in different grades obtained from summer hourly precipitation in Jiulong during 2013-2020.
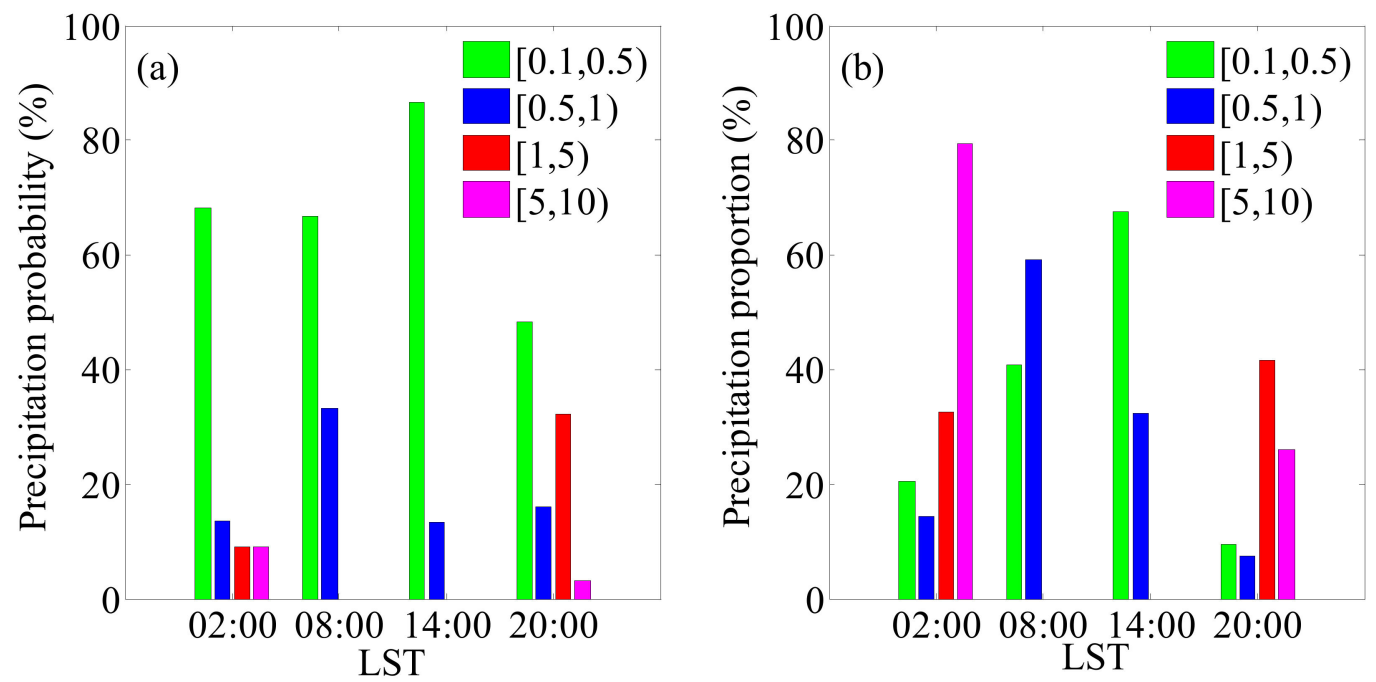

Figure 3. (a) Precipitation probability and (b) precipitation proportion in different grades at launch times in Jiulong from 2013-2020. 


\subsection{Thermal and Dynamic Vertical Structures of Summer Hourly Precipitation}

The vertical distributions of atmospheric temperature, humidity and wind as well as their variations are the reflection of atmospheric thermal and dynamic processes [23]. The above analysis in Jiulong confirms a striking phenomenon in the TP, where most rainfall occurs at night, which is related to the relatively high humidity and strong convective clouds found at night over the TP [24]. The intensive radiosonde observations in Jiulong provide a chance to investigate the atmospheric thermal and dynamic vertical structures of summer hourly precipitation over the TP. In order to avoid the influence of previous precipitation on the current atmospheric thermal and dynamic vertical structures, observations with precipitation in the previous hour were removed, and the sample numbers of hourly precipitation in different grades at launch times in Jiulong from 2013-2020 were obtained (see Table 1 ). For $[1,5) \mathrm{mm}$ grade, the 2 cases at 02 LST had hourly precipitation of 1.9 and $2.4 \mathrm{~mm}$, respectively, while 8 of 10 cases at 20 LST had hourly precipitation of $1-2 \mathrm{~mm}$ and the other 2 cases had hourly precipitation of $2-3 \mathrm{~mm}$. For $[5,10) \mathrm{mm}$ grade, the 2 cases at 02 LST had hourly precipitation of $5-6 \mathrm{~mm}$, and the one case at 20 LST had hourly precipitation of $10.9 \mathrm{~mm}$. The following study is based on the samples in Table 1 .

Table 1. The sample numbers of hourly precipitation in different grades at launch times in Jiulong during 2013-2020 after removing observations with precipitation in the previous hour.

\begin{tabular}{cccccc}
\hline Launch time/LST & $\mathbf{0} \mathbf{~ m m}$ & {$[\mathbf{0 . 1}, \mathbf{0 . 5}) \mathbf{~ m m}$} & {$[\mathbf{0 . 5}, \mathbf{1}) \mathbf{m m}$} & {$[\mathbf{1 , 5} \mathbf{~} \mathbf{m m}$} & {$[\mathbf{5 ,} \mathbf{1 0}) \mathbf{~ m m}$} \\
\hline 02 & 180 & 15 & 3 & 2 & 2 \\
08 & 227 & 10 & 5 & 0 & 0 \\
14 & 269 & 13 & 2 & 0 & 0 \\
20 & 191 & 15 & 5 & 10 & 1 \\
\hline
\end{tabular}

\subsubsection{Vertical Structure of Temperature}

Figure 4 shows the atmospheric temperature profiles in different precipitation grades at launch times in Jiulong from 2013-2020. The background (i.e., no precipitation) atmospheric temperature profile (black line) presents a diurnal variation, in which the temperature is lower at 02 and 08 LST and higher at 14 and 20 LST, showing a response to solar radiation which is more obvious near the surface layer. Under precipitation conditions, the temperature profiles for hourly precipitation within $1 \mathrm{~mm}$ generally present a cold bias to the background atmospheric temperature profile, but hourly precipitation above $1 \mathrm{~mm}$ generally present a warm bias. This may indicate that precipitation with intensity less than $1 \mathrm{~mm} / \mathrm{h}$ is mainly caused by cold air advection, while precipitation with intensity greater than $1 \mathrm{~mm} / \mathrm{h}$ is mainly caused by warm air convection in the lower layer. Considering air convection is caused by atmospheric instability the greater the vertical gradient of atmospheric temperature the more unstable the atmosphere is. Therefore, the temperature gradient for precipitation intensity greater than $1 \mathrm{~mm} / \mathrm{h}$ at 20 and $02 \mathrm{LST}$ is greater than that of the background atmosphere. Note that although the warm air convection mainly caused by solar radiation exists during the day, it hardly results in precipitation due to the dry air in low level during the day over the TP. However, at nighttime the low level air is wet, and the air convection caused by dynamic uplift of terrain can result in precipitation [25]. Moreover, the deviation between precipitation and background atmospheric temperature profiles partly depends on precipitation intensity. This is especially true at 20 LST, where the deviation for precipitation intensity above $5 \mathrm{~mm} / \mathrm{h}$ is greater than that for precipitation intensity within $5 \mathrm{~mm} / \mathrm{h}$. This presents a complex variability of atmospheric thermal stratification over the $\mathrm{TP}$, indicating that condensation latent heat is released in addition to solar radiation and the underlying surface [7]. The influence of these factors on atmospheric thermal stratification over the TP needs to be further studied. Additionally, the deviation between precipitation and background atmospheric temperature profiles is larger in low level than in high level, but the maximum deviation does not appear near the surface layer because of the inversion layer, which is consistent with the results of $\mathrm{Lu}$ and $\mathrm{Li}$ [18]. 

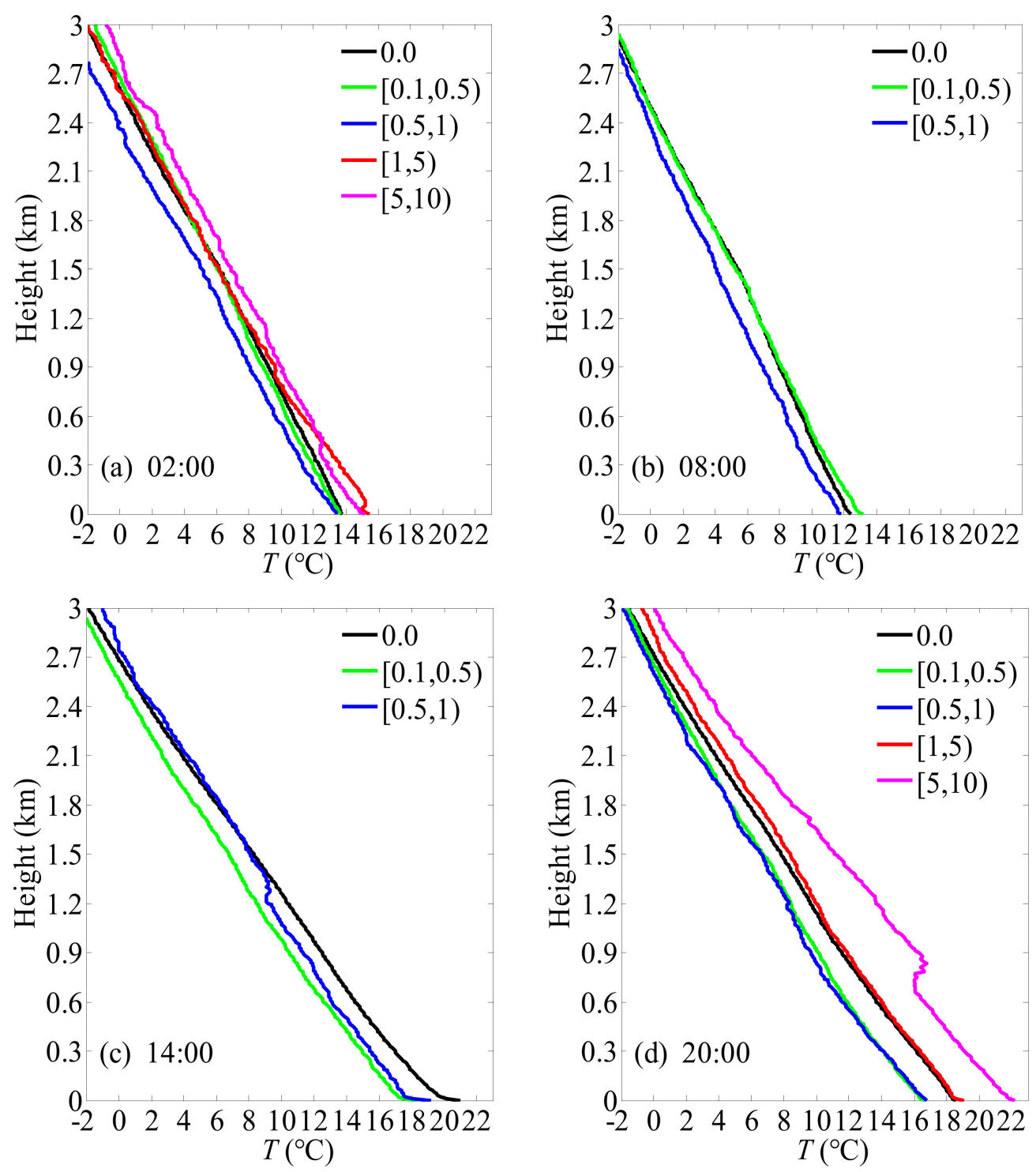

Figure 4. Atmospheric temperature profiles in different precipitation grades at launch times in Jiulong from 2013-2020. (a) 02 LST, (b) 08 LST, (c) 14 LST and (d) 20 LST.

\subsubsection{Vertical Structure of Humidity}

The background atmospheric humidity profile at launch times in Jiulong from 2013-2020 also presents a pattern of diurnal variation. As shown in Figure 5, background atmospheric specific humidity is lower in the daytime and higher at nighttime.This is consistent with the observation obtained by ground-based microwave radiometer in Ganzi (approximately $330 \mathrm{~km}$ northwest of Jiulong), which shows the air is warm and dry during the daytime while cool and wet at nighttime [25]. In general, the specific humidity profile under precipitation presents a wet bias to that of background atmosphere and the bias generally increases with precipitation intensity, indicating that water vapor supply is an important factor affecting precipitation intensity. Moreover, the higher the precipitation grade, the stronger the vertical fluctuation of the corresponding specific humidity profile, especially for hourly precipitation greater than $1 \mathrm{~mm}$ at nighttime. This may imply that the dynamic uplift of terrain plays an important role in nighttime precipitation. All the specific humidity profiles show that specific humidity is generally higher near the ground and decreases gradually with height. However, below the inversion layer there is an inverse humidity layer which is consistent with [18]. This phenomenon occurs because when there is an inversion layer in the atmosphere, the atmospheric stratification state is generally very stable, and this stable structure can make a 
large amount of water vapor stay in the inversion layer and accumulate to form an inverse humidity layer [26]. Similar to temperature profiles, the deviation of a specific humidity profile between precipitation and background atmosphere is larger in low level than in high level, but the maximum deviation does not appear near the surface layer because of the existence of the inversion humidity layer.
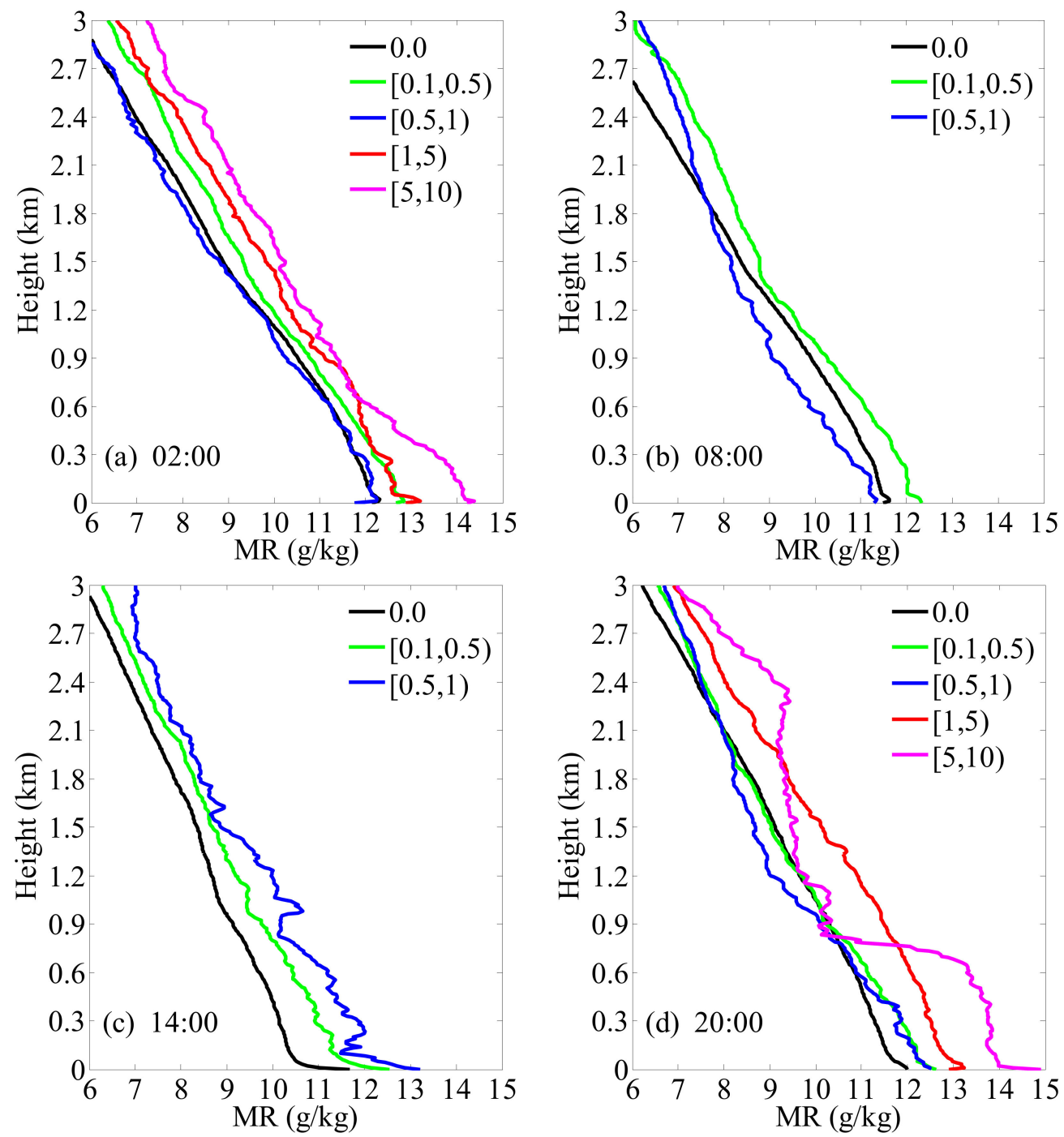

Figure 5. Atmospheric specific humidity profiles in different precipitation grades at launch times in Jiulong during 2013-2020. (a) 02 LST, (b) 08 LST, (c) 14 LST and (d) 20 LST.

\subsubsection{Vertical Structure of Horizontal Wind}

Due to the strong solar radiation and dynamic uplift of terrain especially in a valley area, the atmospheric convection over the TP presents obvious diurnal variation. In early morning, the convective activity is shallow, and the deep convective activity appears in the afternoon. At at night, the convective activity is also strong, which makes it easy to form precipitation clouds, resulting in nighttime precipitation [24]. The fluctuation of the wind speed profile is a reflection of atmospheric convective activity, as shown in Figure 6, where the horizontal wind speed profile at launch times in Jiulong from 2013-2020 also presents such diurnal variation. For background atmosphere (black line), the fluctuation of the wind speed profile is relatively small at 08 LST and large at 14 LST and largest at 20 LST, until at 02 LST the fluctuation is weakened but still larger than that at 08 LST. Moreover, the wind speed profile under precipitation condition also presents the same diurnal variation, and the greater the precipitation intensity, the larger the fluctuation, especially at nighttime 
when the fluctuation of hourly precipitation greater than $1 \mathrm{~mm}$ is more significant. In addition, no low-level jet is found in the horizontal wind speed profile below $3 \mathrm{~km}$ at Jiulong, and the variation of wind speed with height is complex and presents a multi-peaks distribution especially under nighttime precipitation condition. This confirms a remarkable feature of ABL over the TP, which is that the wind speed of ABL over the plateau has a characteristic of multi-level variation and does not increase monotonously with height [24]. Combined with the analysis of temperature and humidity profiles (Figures 4 and 5), this kind of wind field can mix atmospheric temperature more evenly but has less effect on atmospheric humidity homogenization. Li et al. [27] studied the wind field structure of ABL in Gaize of the TP and obtained the same conclusion.
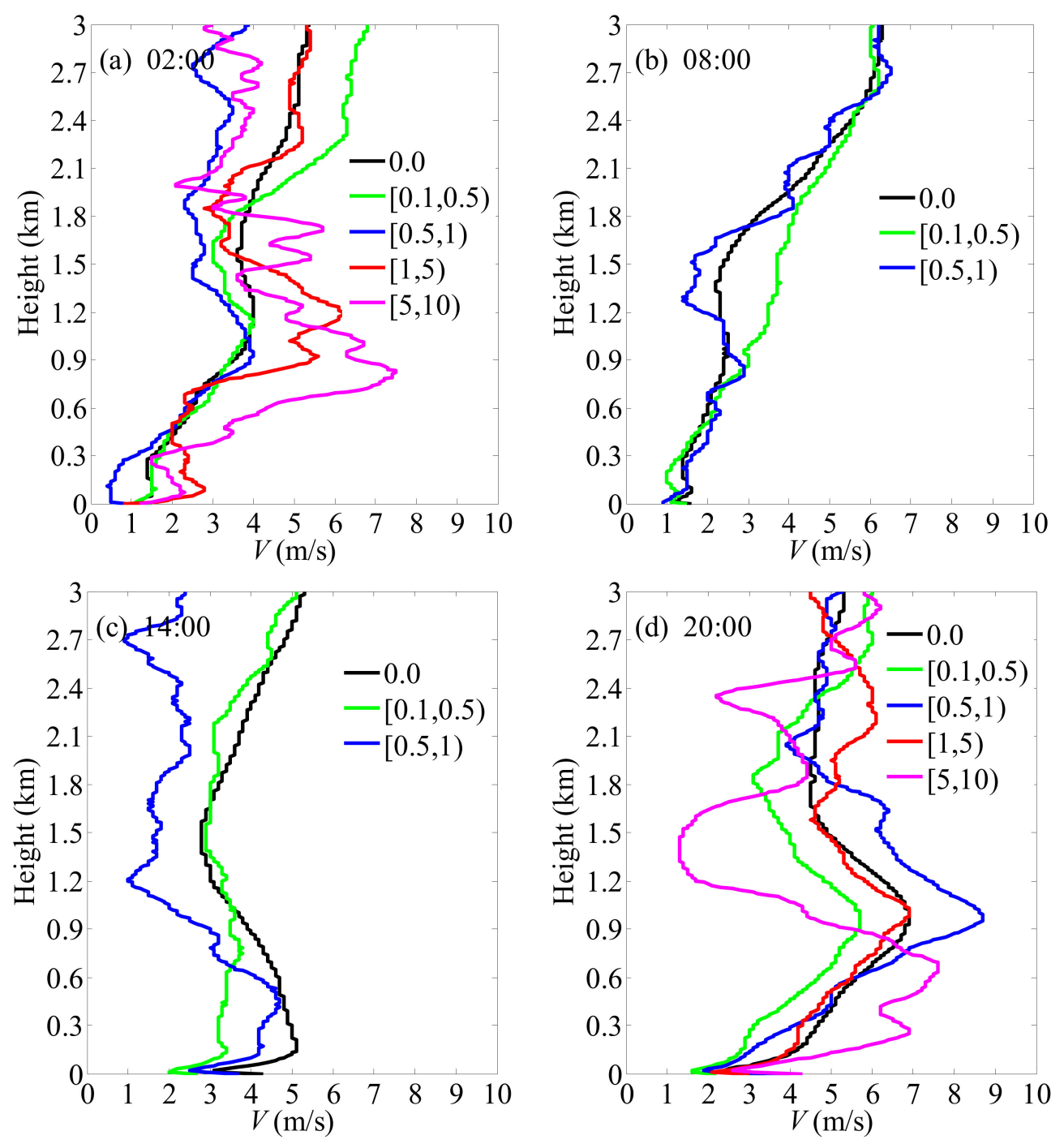

Figure 6. Atmospheric horizontal wind speed profiles in different precipitation grades at launch times in Jiulong from 2013-2020. (a) 02 LST, (b) 08 LST, (c) 14 LST and (d) 20 LST.

The distribution of wind direction is closely related to the terrain. On the one hand, the uneven solar radiation received by the ground is caused by complex terrain, which leads to a local rise and fall of air flow. On the other hand, the fluctuation and slope of terrain can change the direction of air flow near the ground [28,29]. The underlying surface of the TP is mostly continuous mountains. Although the Jiulong observation field is a flat underlying surface, it is a small area surrounded by mountains, so the wind direction is complex and variable. Figure 7 presents the atmospheric horizontal wind direction probability below $3 \mathrm{~km}$ in different precipitation grades at launch times in Jiulong from 
2013-2020. The wind direction has a diurnal variation and precipitation intensity partly depends on wind direction. The prevailing wind direction tends to be northwest for hourly precipitation less than $1 \mathrm{~mm}$ and southwest for that above $1 \mathrm{~mm}$, indicating that the warm and wet southwest airflow is an important condition for producing strong precipitation. Although the prevailing wind direction is northwest during the day, it is complex at night. At night, the prevailing wind direction is northwest for hourly precipitation less than $0.5 \mathrm{~mm}$ and southwest for that greater than $5 \mathrm{~mm}$, while for precipitation of $[0.5,1) \mathrm{mm}$ and $[1,5) \mathrm{mm}$ it is southeast at $02 \mathrm{LST}$ and northwest at $20 \mathrm{LST}$, indicating the variability of wind direction over the TP [24]. Note that the prevailing wind direction of west during the day, including northwest and southwest directions, is consistent with the result of $\mathrm{Lu}$ and Li [18].
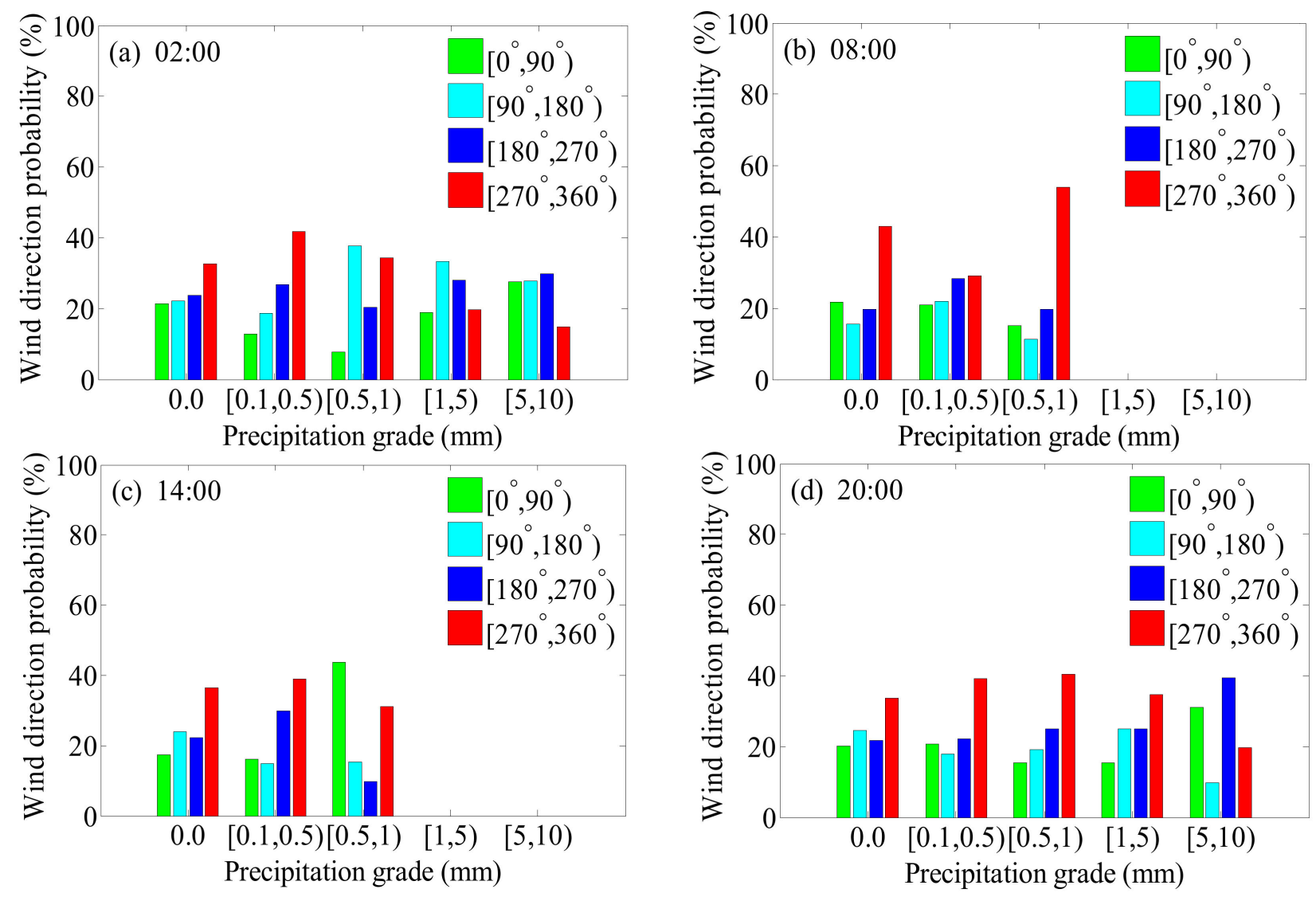

Figure 7. Atmospheric horizontal wind direction probability below $3 \mathrm{~km}$ in different precipitation grades at launch times in Jiulong from 2013-2020. (a) 02 LST, (b) 08 LST, (c) 14 LST and (d) 20 LST.

\section{Discussion}

The weather and climate of the TP is closely related to its high terrain. Due to the strong heating of solar radiation during the day and serious cooling of ground radiation at night caused by the TP terrain, the atmosphere over the TP is warm and dry during the day and cool and wet at night. This leads to clouds occurring more frequently at night than during the day, which then results in the remarkable phenomenon of nighttime precipitation over the TP $[24,25,30]$. Jiulong is located in the eastern TP slope and is a main source of the southwest vortex, with its summer hourly precipitation presenting a significant diurnal variation. Our results show that the precipitation amount, frequency and intensity in Jiulong are higher at night than during the day. The analysis of hourly precipitation in Jiulong during the southwest vortex observation experiment also shows that precipitation greater than $1 \mathrm{~mm} / \mathrm{h}$ mainly occurs at night. High precipitable water and cloud liquid water at night over the TP provide a favorable atmospheric environment for 
nighttime precipitation $[25,31]$. Note that because Jiulong station is on the low mountain side of a river valley with high mountains on two sides, the valley wind caused by the local terrain may play an important role in nighttime precipitation, because the ascending branch of night valley wind circulation can enhance local precipitation [22]. The phenomenon of nighttime precipitation over the TP is different from the precipitation variation over central and eastern China, where the diurnal variations of precipitation amount and frequency show a bimodal feature, with the peaks occurring at 16-17 LST and 06-07 LST, respectively [20].

Diurnal variation of precipitation is a result of the comprehensive influence of atmospheric thermal and dynamic processes on the water cycle, involving the complex interaction between various components of the earth's climate system and a variety of physical processes with different spatial and temporal scales [16]. With analysis on sounding profiles in different precipitation grades in Jiulong, it is found that weak precipitation during the day may be mainly caused by cold air advection, and the strong precipitation at night may be mainly caused by warm air convection in the low layer due to the dynamic uplift of terrain. Moreover, the atmospheric humidity profile and wind profile are sensitive to atmospheric convective activity with reflection through the fluctuation on its profile. Our results show that the higher the precipitation grade, the stronger the fluctuation of specific humidity profile, which is more significant for the wind speed profile. The fluctuation of the wind speed profile presents a diurnal variation, which is small in the morning, large at noon and largest at night, indicating the diurnal cycle of convective activity over the $\mathrm{TP}$, a cycle of convective activity which is conductive to nighttime precipitation [24]. Due to the uneven solar radiation received by the ground and the fluctuation and slope of terrain, wind direction below $3 \mathrm{~km}$ in Jiulong is complex and variable. In general, there is a prevailing wind direction of west during the day which is consistent with [18].

Both our study and $\mathrm{Lu}$ and $\mathrm{Li}$ [18] confirm that there is an inverse humidity layer below the inversion layer when summer precipitation occurs in Jiulong. The formation of an inverse humidity layer is complex because there are many influencing factors. It is generally believed that in addition to the inversion layer, other factors such as atmospheric advection transport, weather change and underlying surface can also contribute to the formation of inverse humidity [32,33]. The phenomenon of the inversion layer accompanied by an inverse humidity layer over the TP needs further study to reveal its formation mechanism. Additionally, the wind speed of ABL under precipitation condition in Jiulong does not increase monotonously with height and presents multi-peaks distribution. This kind of wind field is also a remarkable feature of ABL over the TP [24,27]. The multi-level variation in the wind field is complex because it may be related to a strong baroclinic formed by a horizontal temperature gradient, strong buoyancy effect of turbulent motion and dynamic suction pump of ABL over the TP [24,32]. Our results reveal the characteristics of summer precipitation on subdiurnal scale in Jiulong and exhibit the observational facts on atmospheric thermal and dynamic vertical structure for summer hourly precipitation. Since the precipitation over the TP is complex due to its unique terrain, to fully understand the atmospheric phenomenon over the TP, more studies need to be done on physical mechanisms, especially with the aid of numerical weather models.

\section{Conclusions}

Using the intensive sounding profiles of the southwest vortex observation experiment in Jiulong and the hourly precipitation from June to August 2013-2020, the characteristics of summer precipitation on a subdiurnal scale were analyzed, and the atmospheric thermal and dynamic vertical structures in different precipitation grades were also investigated. The conclusions obtained are as follows.

Summer hourly precipitation in Jiulong shows a diurnal variation especially for precipitation greater than $1 \mathrm{~mm} / \mathrm{h}$. Both precipitation amount and precipitation frequency present a unimodal pattern with low value during the day and high value at night. A bimodal pattern is found in precipitation intensity for hourly precipitation greater than 
$1 \mathrm{~mm}$, with the main peak at 22 LST and the second peak at 09 LST. The analysis on hourly precipitation at launch times in Jiulong also shows that the hourly precipitation during the day is generally less than $1 \mathrm{~mm}$ and greater than $1 \mathrm{~mm}$ mostly occurs at night, resulting in a high proportion of nighttime precipitation. Besides high precipitable water and cloud liquid water, the dynamic effect of local terrain is also conducive to the occurrence and enhancement of nighttime precipitation.

Compared to the temperature profile of the background atmosphere in Jiulong, the temperature profile for hourly precipitation less than $1 \mathrm{~mm}$ is cold. The weak precipitation during the day may be mainly caused by cold air advection, and the strong precipitation at night may be closely related to warm air convection in the low layer due to the dynamic uplift of terrain. The deviation between precipitation and background atmospheric temperature profiles partly depends on precipitation intensity.

The humidity profile under precipitation conditions is mostly wet, and the wet bias generally increases with precipitation intensity, indicating that water vapor is an important factor affecting precipitation intensity. Both humidity profile and wind speed profile show obvious fluctuation when precipitation occurs, and the greater the precipitation intensity, the larger the fluctuation, especially for nighttime precipitation. Moreover, the fluctuation of the wind speed profile is small in the morning, large at noon and largest at night, presenting a similar diurnal variation to that of convective activity over the TP which is conducive to nighttime precipitation. Although the wind direction below $3 \mathrm{~km}$ in Jiulong is complex and variable, there is a prevailing wind direction of west during the day.

Some remarkable phenomena are found in the sounding profiles. There is an inverse humidity layer below the inversion layer when summer precipitation occurs in Jiulong. The wind speed of the ABL under precipitation conditions does not increase monotonously with height and presents multi-peaks distribution. These two phenomena tend to be closely related with the underlying surface and topography of Jiulong. Note that this study mainly focused on revealing the observational facts of summer precipitation variation on subdiurnal scale and its atmospheric thermal and dynamic vertical structures in Jiulong. More studies on physical mechanism and numerical simulation are still necessary to better understand the atmospheric phenomenon over the TP.

Author Contributions: Conceptualization, Y.T. and G.X.; methodology, G.X.; software, Y.T.; validation, G.X., R.W. and X.W.; formal analysis, Y.T.; resources, J.W.; data curation, P.L.; writing-original draft preparation, Y.T.; writing-review and editing, G.X.; visualization, Y.T.; supervision, X.W.; project administration, R.W.; funding acquisition, R.W. All authors have read and agreed to the published version of the manuscript.

Funding: This research was funded by the National Natural Science Foundation of China (Grant Nos. 41975058 and 91637211) and The National Key Research and Development Program of China (Grant No. 2018YFC1507201).

Institutional Review Board Statement: Not applicable.

Informed Consent Statement: Not applicable.

Data Availability Statement: Data used in this study can be requested from the authors under the research institute polices of the Institute of Heavy Rain, China Meteorological Administration, Wuhan.

Acknowledgments: We would like to thank the Chengdu Institute of Plateau Meteorology of China Meteorological Administration and the National Meteorological Center of China for providing the data used in this study.

Conflicts of Interest: The authors declare no conflict of interest.

\section{References}

1. Pan, B.; Li, J.J. Qinghai Tibetan Plateau: A driver and amplifier of the global climatic change III: The effects of the uplift of Qinghai-Tibetan Plateau on climatic changes. Lanzhou Univ. J. Nat. Sci. Ed. 1996, 32, 108-115. (In Chinese) [CrossRef]

2. Zhang, S.L.; Tao, S.Y.; Zhang, Q.Y. Large and meso- $\alpha$ scale characteristics of intense rainfall in the mid- and lower reaches of the Yangtze River. Chin. Sci. Bull. 2002, 47, 779-786. (In Chinese) [CrossRef] 
3. Wu, G.X.; Liu, Y.M.; Liu, X. How the Heating over the Tibetan Plateau affects the Asian climate in summer. J. Atmos. Sci. 2005, 29, 47-56. (In Chinese) [CrossRef]

4. Wang, H.; Liu, G.; Wang, S.; He, K. Precursory Signals (SST and Soil Moisture) of Summer Surface Temperature Anomalies over the Tibetan Plateau. Atmosphere 2021, 12, 146. [CrossRef]

5. Zhao, P.; Chen, L.X. The climatic characteristics of the atmospheric heat source of the Qinghai-Tibet Plateau in the past 35 years and its relationship with precipitation in China. Sci. China (Ser. D) 2001, 31, 327-332. (In Chinese)

6. Xu, X.D.; Zhao, T.L.; Shi, X.F. A study of the role of the Tibetan Plateau's thermal forcing in modulating rainband and moisture transport in eastern China. Acta. Meteor. Sin. 2015, 73, 20-35. (In Chinese) [CrossRef]

7. Yao, X.P.; Zhang, S.; Yan, L.Z. Research progress on the atmospheric heat source over the Tibetan Plateau and its influence. Trans. Atmos. Sci. 2019, 42, 641-651. (In Chinese) [CrossRef]

8. Wang, X.F.; Li, C.H.; Yang, J.Y.; Wang, S.M.; Fu, M.; Yi, L. Research progress on east-moving cloud clusters from the Qinghai-Tibet Plateau. Torrential Rain Disasters 2020, 39, 433-441. (In Chinese) [CrossRef]

9. Zhuo, G.; Xu, X.D.; Chen, L.S. Instability of eastward movement and development of convective cloud clusters over Tibetan Plateau. J. Appl. Meteor. Sci. 2002, 13, 447-456. (In Chinese) [CrossRef]

10. Zheng, Y.G.; Wu, G.X.; Liu, Y.M. Dynamical and thermal problems in vortex development and movement. Part I: A PV-Q view. Acta. Meteor. Sin. 2013, 71, 185-197. (In Chinese) [CrossRef]

11. Ma, T.; Liu, Y.M.; Wu, G.X. Effect of potential vorticity on the formation, development, and eastward movement of a Tibetan Plateau vortex and its influence on downstream precipitation. Chin. J. Atmos. Sci. 2020, 44, 472-486. (In Chinese) [CrossRef]

12. Xu, X.D.; Chen, L.S. Advances of the study on Tibetan Plateau experiment of atmospheric sciences. J. Appl. Meteor. Sci. 2006, 17, 756-771. (In Chinese)

13. Duan, A.M.; Wu, G.X.; Liu, Y.M.; Ma, Y.M.; Zhao, P. Weather and climate effects of the Tibetan Plateau. Adv. Atmos. Sci. 2012, 29, 978-992. [CrossRef]

14. Zhao, P.; Xu, X.; Chen, F.; Guo, X.; Zheng, X.; Liu, L.; Hong, Y.; Li, Y.; La, Z.; Peng, H.; et al. The Third Atmospheric Scientific Experiment for American meteorological society understanding the earth-atmosphere coupled system over the Tibetan plateau and its effects. Bull. Amer. Meteor. Soc. 2018, 99, 757-776. [CrossRef]

15. Zhao, P.; Li, Y.Q.; Guo, X.L.; Xu, X.D.; Liu, Y.M.; Tang, S.H.; Xiao, W.M.; Shi, C.X.; Ma, Y.M.; Yu, X.H.; et al. The Tibetan Plateau surface-atmosphere coupling system and its weather and climate effects: The Third Tibetan Plateau Atmospheric Science Experiment. J. Meteor. Res. 2019, 33, 375-399. [CrossRef]

16. Yu, R.; Li, J. Regional characteristics of diurnal peak phases of precipitation over contiguous China. Acta. Meteor. Sin. 2016, 74, 18-30. (In Chinese) [CrossRef]

17. Li, Y.Q.; Xu, X.D. A review of the research and observing experiment on Southwest China Vortex. Adv. Meteor. Sci. Technol. 2016, 6, 134-140. (In Chinese) [CrossRef]

18. Lu, P.; Li, Y.Q. Analyses of the boundary layer characteristics by intensive sounding observation data at Jiulong station in summer for 9 years. Plateau Meteor. 2020, 39, 1058-1069. (In Chinese) [CrossRef]

19. Steiner, M.; Houze, J.R.; Yuter, S.E. Climatological characterization of three-dimensional storm structure from operational radar and rain gauge data. J. Appl. Meteor. 1995, 34, 1978-2007. [CrossRef]

20. Zheng, Y.G.; Gong, Y.D.; Chen, J.; Tian, F.Y. Warm-Season diurnal variations of total, stratiform, convective, and extreme hourly precipitation over Central and Eastern China. Adv. Atmos. Sci. 2019, 36, 143-159. [CrossRef]

21. Li, J.; Yu, R.; Zhou, T. Seasonal variation of the diurnal cycle of rainfall in the southern contiguous China. J. Clim. 2008, 21, 6036-6043. [CrossRef]

22. Singh, P.; Nakamura, K. Diurnal variation in summer precipitation over the central Tibetan Plateau. J. Geophys. Res. 2009, 114, D20107. [CrossRef]

23. Yu, Y.; Wan, R.; Zhang, W.G.; Zhou, W. Comparative analysis of vertical structure of the atmosphere before and after heavy rainfall in mountain and plain. Torrential Rain Disasters 2020, 39, 354-362. (In Chinese) [CrossRef]

24. Li, G.P. Dynamic Meteorology of the Tibetan Plateau; Meteorology Press: Beijing, China, 2002.

25. Xu, G.R.; Zhang, W.G.; Wan, X.B.; Wang, L.; Leng, L.; Zhou, L.; Wan, R. Analysis on atmospheric profiles retrieved from microwave radiometer observation at Ganzi in the eastern Qinghai-Tibet Plateau. Torrential Rain Disasters 2019, 38, 238-248. (In Chinese) [CrossRef]

26. Li, Z.J.; Jin, L.L.; He, Q.; Miao, Q.L.; Li, M.M. Characteristics of specific humidity distribution and profiles in Urumqi City and suburbs. Arid Zone Res. 2020, 43, 977996. (In Chinese) [CrossRef]

27. Li, J.L.; Hong, Z.X.; Sun, S.F. An observational experiment on the atmospheric boundary layer in Gerze area of the Tibetan Plateau. Chin. J. Atmos. Sci. 2000, 24, 301-311. (In Chinese) [CrossRef]

28. Li, G.P. Progress and prospects in research of mountain meteorology in China during the past 25 years. Adv. Meteor. Sci. Technol. 2016, 6, 115-122. (In Chinese) [CrossRef]

29. Jia, C.H.; Dou, J.J.; Miao, S.G.; Wang, Y.C. Analysis of characteristics of mountain-valley winds in the complex terrain area over Yanging Zhangiiakou in the winter. Acta. Meteor. Sinica. 2019, 77, 475-488. (In Chinese) [CrossRef]

30. Wan, X.; Xu, G.; Wan, R.; Wang, B.; Ren, J.; Luo, C. Vertical structure of non-precipitation cloud obtained from cloud radar observation at Ganzi in the eastern Qinghai-Tibet Plateau. Torrential Rain Disasters 2020, 39, 496-504. (In Chinese) [CrossRef] 
31. Xu, G.; Cui, C.; Li, W.; Zhang, W.; Feng, G. Variation of GPS precipitable water over the Qinghai-Tibet Plateau: Possible teleconnection triggering rainfall over the Yangtze River Valley. Terr. Atmos. Ocean. Sci. 2011, 22, 195-202. [CrossRef]

32. Zhou, M.Y.; Qian, F.L.; Chen, Z.; Li, S.M.; Su, L.R.; Xu, X.D.; Chen, L.S.; Liu, J.C.; Xi, Z.; Zhu, D. The characteristics of the profiles for wind, temperature and humidity in the baroclinic convective boundary layer on Xizang plateau. J. Geophys. 2002, 45, 818-830. [CrossRef]

33. Luo, B.; Zhuo, G.; Yang, X.H. The atmospheric characteristics in the early period of east Asian monsoon in Gaize Tibet. Plateau Mt. Meteorol. Res. 2009, 29, 1-5. (In Chinese) [CrossRef] 